\title{
Value-Oriented IT Project Portfolio Management
}

\author{
Rongzeng Cao ${ }^{1}$, Wei Ding', Chunhua Tian', and Juhnyoung Lee ${ }^{2}$ \\ 1 IBM China Research Lab \\ Beijing, China \\ \{caorongz, dingw, chtian\}@en.ibm.com \\ 2 IBM T. J. Watson Research Center \\ New York, USA \\ jyl@us.ibm.com
}

\begin{abstract}
In the present enterprise computing environment, it becomes more and more important to be able to demonstrate financial gains of IT (Information Technology) initiatives compared to their cost. A valuation methodology is a logical, repeatable framework for making IT investment decisions and monitoring projects to ensure that they ultimately contribute to the financial health and growth of the enterprise. Traditionally, organizations have used a bottom-up approach for technology valuation by using metrics such as return on investment and net present value. While it provides a simple, easy-to-understand valuation mechanism, the traditional approach is limited in the type of analyses. To provide more flexible and versatile valuation of technology, we propose a value-oriented project portfolio management with which organizations can view IT staff and initiatives not only as costs but also as assets managed in a similar way as a fund manager would apply to any other investment. The proposed approach is comprised of multiple steps that collectively compute and help maximizing value of IT initiatives for business transformation. It integrates a value model with a project portfolio to factor in financial values in selecting projects and optimizing the portfolio.
\end{abstract}

\section{Introduction}

In the present enterprise computing environment, it becomes more and more important to be able to demonstrate financial gains of IT (Information Technology) initiatives compared to their cost [1]. Most organizations are constrained by resources to meet their requirements and objectives. Selecting projects to fund poses an important and hard problem faced by CIOs and CTOs of the organizations. It is not as simple as ranking the proposals and funding them from the top of the list until resource is depleted. Organizations need to analyze the business value and balance

Please use the following format when citing this chapter:

Cao, R., Ding, W., Tian, C., Lec, J., 2006, in International Federation for Information Processing, Volume 205, Rescarch and Practical Issues of Enterprisc Information Systems, eds. Tjoa, A.M., Xu, L., Chaudhry, S., (Boston:Springer), pp.219-229. 
the benefits that project portfolios provide with their respective constraints, and they need to do so in a meaningful way [2]. To meet this requirement, organizations need a valuation methodology, which provides a logical, repeatable framework for making IT investment decisions and monitoring projects to ensure that they ultimately contribute to the financial health and growth of the enterprise.

Traditionally, organizations have used a bottom-up approach for technology valuation. First, they would define financial metrics such as ROI (Return on Investment), NPV (Net Present Value), IRR (internal rate of return), pay-back period, etc $[3,4]$. Then, they use the metrics to evaluate candidate initiatives. The most significant limitation of using the traditional approach to project prioritization is that they provide, at best, only a partial representation of what is relevant. Financial metrics, quite simply, capture the objectives of an organization only partially. There are non-financial, intangible objectives in organizations such as customer satisfaction and brand image. To provide more flexible and versatile valuation of technology, we propose a value-oriented project portfolio management with which organizations can view IT staff and projects not only as costs but also as assets managed in a similar way as a fund manager would apply to any other investment.

The rest of this paper is structured as follows: Section 2 presents the valuation framework of the proposed value-oriented IT project portfolio management. The following sections describe each step of the value-oriented project portfolio management framework in detail, and explain their analysis methods, with examples. Sections 3, 4, 5, and 6 present analyses using component business modeling, solution similarity measures, value driver trees, and portfolio analysis algorithms, respectively. Section 7 summarizes previous work, and discusses how the presented approach is different. In Section 8, conclusions are drawn and future work is outlined.

\section{The Framework}

Based on our study and practice, a value-oriented project portfolio management methodology is formed and proposed to facilitate the end-to-end business process transformation planning from gap analyses to portfolio management as shown in Fig. 1. The proposed methodology provides a top-down approach to analyzing the business value of technology initiatives. The objectives of approach include providing visibility into project performance, helping manage a dynamic portfolio of business transformation initiatives, and monitoring financial metrics for transformation initiatives and projects. The framework consists of the four modules that are linked together.

The proposed approach starts with a business transformation analysis by using Component Business Modeling (CBM) which enables enterprise strategic design and planning. This step utilizes the CBM technique to understand the business and organization and identify business transformation opportunities. The CBM approach is useful in determining which solutions and projects to consider, and assuring that investments are classified properly and diversified across various business processes. 
The gap analysis based on CBM helps the organization assess rigorously its enterprise systems and processes, and identify opportunities for improvement. Also, this top-down approach enables a business analysis for identifying "hot" components that are associated with business pain points, and various types of IT shortfalls that need to be addressed to mitigate the pain points.

Then, the solution analysis step helps discover which solution or solution portfolio in a given solution catalog supports the processes improvement opportunities identified in the previous step. A solution catalog presents a set of complete business and technology solutions for an industry in a process-oriented way.

In the next step, a value model is developed to identify and map the enterprise's key business and IT value drivers, and links them to the measurable business and financial benefits. Specifically, it quantitatively calculates the impact of multiple business initiatives at a time such as solutions to the performance of business activities and then to key performance indicators of the organization, through the cause and effect relationships over time.

Furthermore, it integrates a value model with a project portfolio to factor in financial values in selecting projects and optimizing the portfolio. Project portfolio optimization supports provision of decision support for selecting candidate investments, prioritizing transformations initiatives and projects based on their potential to improve business performance, maximizing the value delivered by initiatives and at the same meet constraints such as budget and resource, and balancing the overall portfolio with enterprise objectives.

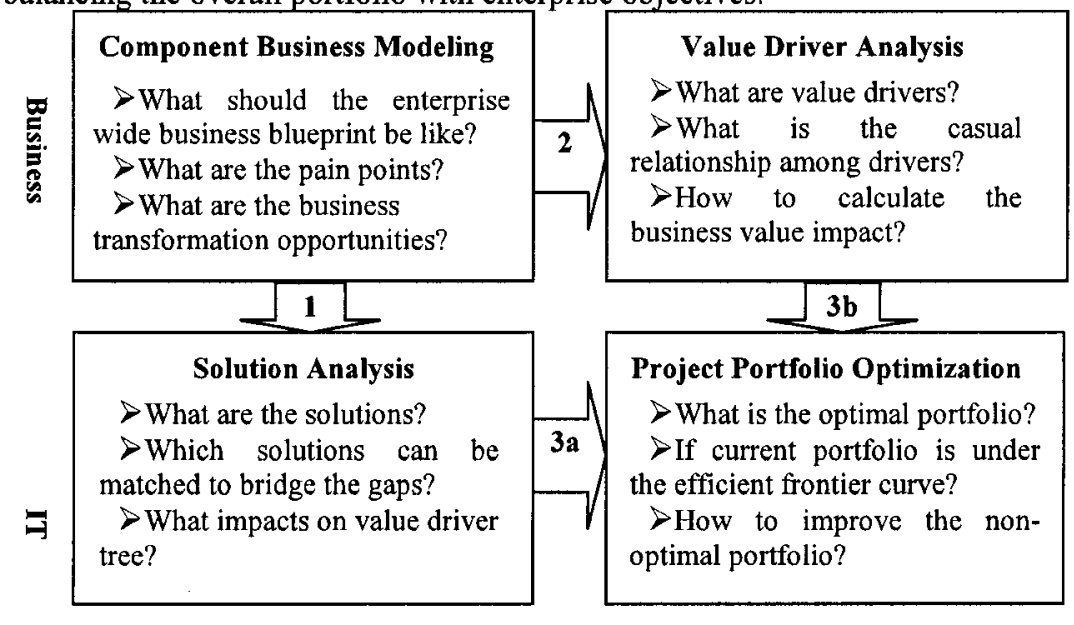

Enterprise Level Focus

Project Level Focus

Fig. 1. Value-oriented project portfolio management 


\section{Component Business Modeling}

To help determine which projects to consider, and to ensure that investments are classified properly and diversified across various business processes, we utilize the Component Business Modeling. CBM is a business decomposition methodology that has been used successfully by IBM in various engagements across industry sectors $[5,6]$.

CBM models a business as a set of business components. A business component is a part of an enterprise that has the potential to operate independently, in the extreme case as a separate company, or as part of another company. A business component is a logical view of part of an enterprise that includes the resources, people, technology and know-how necessary to deliver some value.

A business component map is a tabular view of the business components in the scope of interest. The columns of the table represent business competencies and the rows represent accountability levels. The business components are rectangles within the table. Normally each rectangle is within only one cell of the table. Fig. 2 shows a sample component business model for the banking industry.

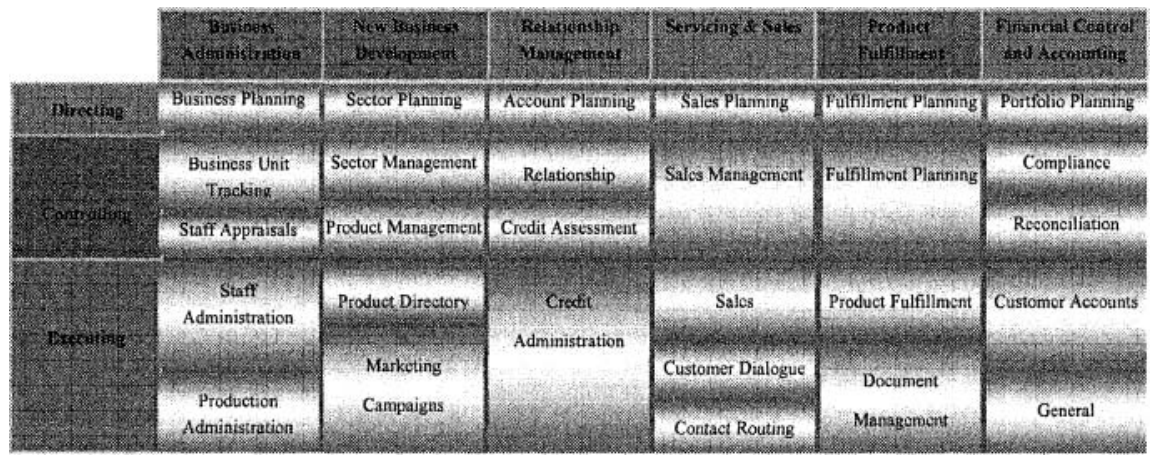

Fig. 2. A component business model

Once a business component map is created, an IT shortfall assessment can be conducted to examine each key business component and then each business process within the component for improvement opportunities. Within each key component, the current systems, processes and organizations are specified. In addition, there may be more detailed specifications on the functional and non-functional features of the business components and the collaborations among components. The systems, processes and organizations can be analyzed for the cost effectiveness and business efficiency of the current state ("As-Is") and the future state ("To-Be"). The shortfall analysis of the business component efficiency and effectiveness determines if a certain technology-based reengineering exercise can help streamline the process, improve operating efficiency and optimize strategic business advantage. Generally, three types of issues tend to arise in the shortfall assessment:

Gaps: no system exists, the system lacks key functionality, or is poorly designed/uses the wrong technology for a specific component. 
Duplication: multiple systems compete for the same component, typically adding unnecessary complexity/cost to development, maintenance and production.

Over-extension: a system designed to support one component is extended to help support others, for which it may not have appropriate capabilities. Furthermore, as a system gets more extensive the cost/complexity of its operation increases exponentially.

\section{Solution Analysis}

A solution catalog presents a set of business solutions for an industry in a processoriented way. A solution catalog tree for banking industry is shown in Fig. 3.

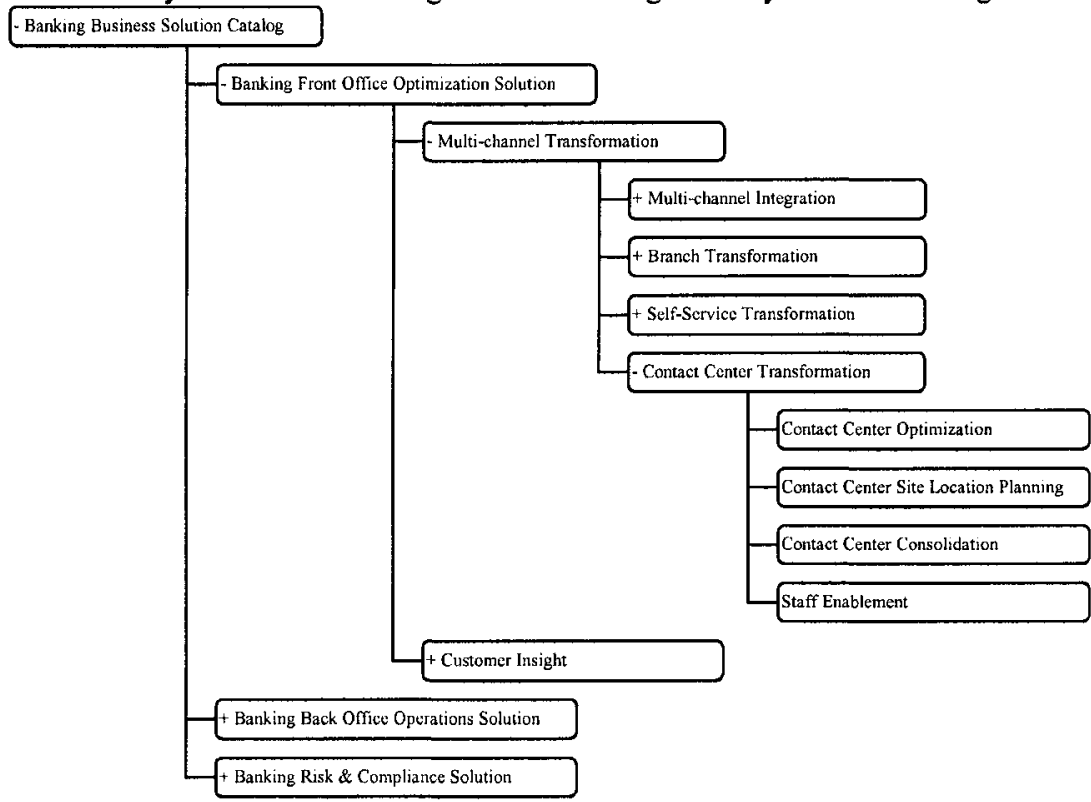

Fig. 3. A solution catalog

We use business processes within an organization to link solution technologies with the CBM map and show how various business components and processes are covered by solutions. It is designed to help visualize, plan, and implement a coherent, integrated, and comprehensive information technology solutions for an organization or enterprise. The solution catalog is represented hierarchically by a tree structure. The top level nodes represent business solution categories, the middle level nodes business solution offerings, and the leaf nodes business solution components and linkages.

The previous step of the CBM shortfall assessment identified various technology gaps in the present enterprise environment. By using the specifications of the solution catalog, this step identifies one more existing solutions that best address the 
shortfalls identified in the previous step, through a matching component. In order to determine the basic set of matching solutions, we compute the degree of similarity between feature values (including, but not limited to, key business metrics for improvement, e.g., number of employees, implementation costs, etc.) of the matching component and those of the solutions from the solution catalog by using a distance-based similarity measure.

Although building a complete solution catalog is costly in terms of time, money and skills involved. In fact, it needn't have the value of completely vendorindependent solution catalogs. So it can not only be determined and maintained efficiently by major consulting companies. Solution catalog is useful way to help organizations to view industry solutions that are specific to their business and paint points, plan, and implement a comprehensive, integrated, and coherent solution.

\section{Value Driver Analysis}

Traditionally, organizations have used a bottom-up approach for technology valuation. The traditional ROI analysis defines financial metrics such as NPV (Net Present Value), IRR (internal rate of return), and pay-back period. The traditional approach is hard to generalize and costly to evaluate multiple initiatives at one time, provide a complete picture of the impact of these solutions or initiatives, and determine which solutions are best.

To address this problem, our approach proposes to link characteristics and impacts of proposed solutions and initiatives to a value model, which is typically represented by a top-down structure called a value driver tree. It captures business impact at a measurable metric level and aggregates it into higher-level business values by using generic value drivers such as revenue growth, margin improvement, and increased capital efficiency.

The value driver tree provides a hierarchical structure of value drivers and metrics. The top level nodes of the value driver tree are generic value driver, such as the shareholder value or profitability; the middle level nodes are business specific value driver; and the leaf nodes are measurable operational metrics. The leaf metrics nodes are connected to the root through multiple layers of performance indicators and value drivers. A sample value driver tree is shown in Fig. 4. 


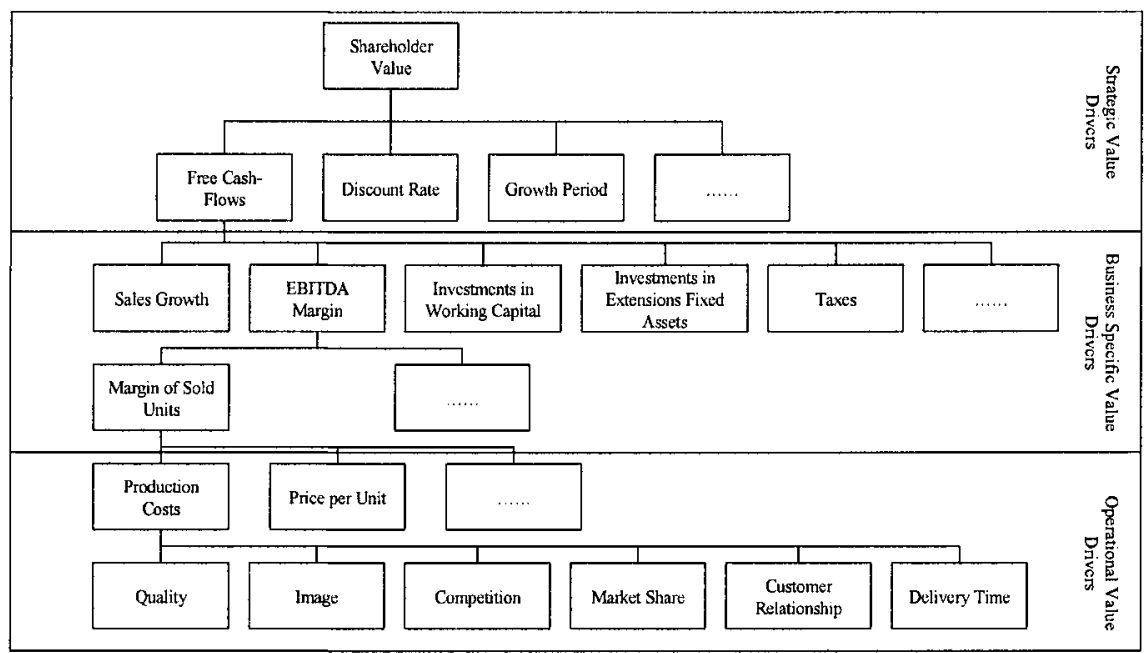

Fig. 4. A value driver tree

Before using a value driver tree to calculate the business value of one or more solutions, the causal relationship between a value driver and its children value drivers needs to be defined first. In certain cases, it is difficult to come up with quantitative relationship among a value driver node and its children nodes, because empirical data for applying regression methods is not always available. Our approach proposes a new technique referred to as " $1 \%$ sensitivity analysis." The $1 \%$ sensitivity analysis is an incremental expression form to describe the impact of one metrics (cause) on another. For example, if a metric, order process time, is reduced $1 \%$, this analysis computes how much change there will be to another metric, say, revenue. Domain experts can come up with this data by using their experience and estimation.

Because the value model is built on causal judgment, the model's assumptions must be clearly specified, so that they can be discussed, debated, and set to reflect best-organizational understanding of how the organization determines the value it creates. In a real-world situation, the value driver tree can usually be constructed in a 2-3 day framing workshop using techniques based on influence diagramming, and causal modeling.

Based on the $1 \%$ sensitivity analysis, a recursive algorithm is used to calculate the expected values of high-level value drivers affected by low-level value drivers or solutions. The value of driver $y$ is:

$$
\mathrm{y}_{10+1}=\mathrm{y}_{\mathrm{t} 0} \times \Delta_{\mathrm{y}}
$$

where $\Delta_{y}=\sum \Delta_{y}{ }^{(x i)}$ is the $1 \%$ sensitivity of $y . \Delta_{y}{ }^{\left({ }^{(x i)}\right)}$ is the $1 \%$ sensitivity between the parent value driver $y$ and one of its children value driver $x_{i}, i=1,2,3, \ldots n . n$ is the number of children value drivers.

The recursive algorithm forecasts the planned solution portfolio's impact on value drivers. This activity can be repeated for a number of alternative solution portfolio candidates. It helps determine the highest-reward, lowest-risk solutions, set priorities and ensure proper resource allocation. 


\section{Portfolio Analysis}

After one or more solutions are selected and evaluated by the solution matching and the value analysis, there could be several project candidates. Most organizations are constrained by resources to meet their requirements and objectives. Selecting projects to fund poses an important and hard problem. It is not as simple as ranking the proposals and funding them from the top of the list until resource is depleted. To address this problem, we use an enterprise portfolio analysis to select the optimal project mix to maximize the collective benefit, while balancing other factors such as risk, dependency, and budget. A mathematical model based on the knapsack problem [7] together with a revised dynamic programming algorithm is developed.

A standard mathematical model of the portfolio optimization problem is constructed as follows: Let $P$ denote the set of available projects. For now, assume that the projects are independent. That is, it is reasonable to select any combination of projects and the cost and benefit of any project do not depend on what other projects are selected. Define, for each project $i$ in the available set $P$ (denoted $i \in P$ ), the zero-one variable $x_{i}$. The variable $x_{i}$ is one, if the project is accepted, and zero, if it is rejected. Let $b_{i}$ be the benefit of the ith project and $c_{i}$ be its cost. Let $C$ be the total available budget. The goal is to select from the available project set $P$ a subset of projects with a total cost less than or equal to $C$ that produces the greatest possible total benefit. The problem may be mathematically expressed as:

\section{Maximize $\sum b_{i} x_{i}$ \\ Subject to: $\sum \mathrm{c}_{\mathrm{i}} \mathrm{x}_{\mathrm{i}} \leq \mathrm{C}, \mathrm{x}_{\mathrm{i}}=0$ or 1}

where the notation $\sum$ denotes summing over all projects $i$ in $P$.

Although the basic model is simplistic, it can be extended to handle many complexities that must be addressed in the real world. In real-world practices, the model has been extended to deal with interdependencies among projects, multiperiod, and sensitivity to delay, risk, side-effects (e.g. the introduction of a new $\mathrm{CRM}$ solution might require additional organizational restructuring in other areas, too) etc. Here let is just take interdependencies as an example to show an extension. Examples of dependencies that may exist between projects include: project $A$ is not funded unless project $B$ is funded, both project $A$ and $B$ must be funded or neither is funded, and funding project $A$ precludes funding project $B$. By adding constraints to the basic model, interdependencies among projects can be handled by groups. Groups mean alternatives can be grouped with constraints. Suppose $G$ denotes a group of the projects with some constraints, the following constraints can be added:

$\sum x i \leq 1, i \in G$ (Less than or equal to 1 ) - at most one alternative in a group can be funded.

$\sum x i=1, i \in G$ (Equal to 1 ) - one and only one alternative in a group must be funded.

$\sum x i \geq 1, i \in G$ (Greater than or equal to 1 ) - one or more alternatives in a group must be funded.

Based on this model, a revised dynamic programming algorithm is used to pick possible project portfolios that create the greatest possible risk-adjusted value without exceeding the applicable constraints on available resources. The set of investments that create the greatest possible value at the least possible cost are the 
"efficient frontier" [8]. Fig. 5 shows an example of 50 projects in a company with the efficient frontier. It lists all possible portfolios and the optimal portfolio at different given budget (i.e., cost). Portfolios along the curve are said to be efficient because the company is getting the maximum value from the available budget. Points under the efficient frontier curve represent inefficient portfolios.

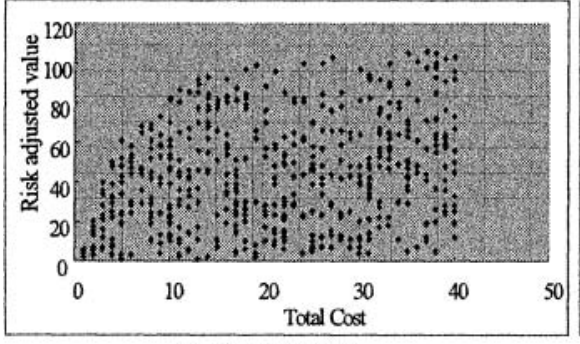

All Possible Portfolios

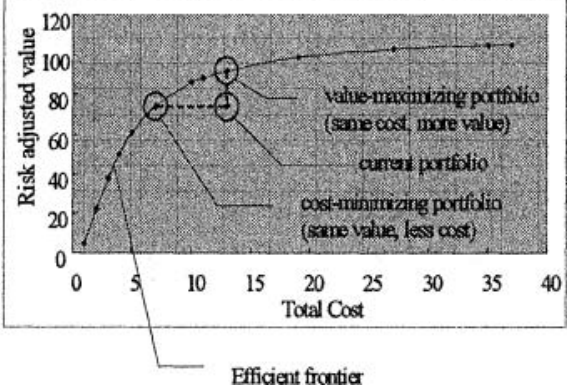

Fig. 5. Portfolio analysis and optimization

The company can also review the current portfolio of projects on or under the efficient frontier. There are two ways to improve points under the efficient frontier curve. One is value-maximizing portfolio (same cost, more value), and the other is cost-minimizing portfolio (same value, less cost). In short, the portfolio analysis and optimization can review the current portfolio and deliver the greatest margins, maintain an optimal portfolio mix considering the overall risk.

\section{Related work}

There are precursory thoughts on value-oriented IT project portfolio management found in research reports and published papers $[9,10]$. For example, Tom Pisello has developed a methodology called IT Value Chain Management to optimize measures and manage the business impact of IT for all stakeholders based on their research findings on the correlation between IT spending and shareholder value. IT Value Chain Management approach quantifies technology benefits, and improves the financial justification process for technology investments, and calculates the value of specific business and IT capabilities such as faster time-to-market [11].

Also, a Forrester research report discusses "installing value-based thinking." The report describes that a strategic IT organization contributes, directly and indirectly, to the organization's ability to execute its business plan. Therefore, strategic thinking for the IT manager must include an analysis of the impact that major decisions will have outside the IT organization. This focus must be driven down through the organization so that the business implications of decisions are considered at all levels. The research firm also provides related discussion on the application of balanced scorecard for IT and value metrics for IT along with ROI analysis of IT [9]. 
In addition, there are numerous studies on return-on-investment, metrics measurement and analysis models. Total Cost of Ownership Model by the Gartner Group provides a deterministic ROI model for calculation designed to help consumers and enterprise managers assess direct and indirect costs as well as benefits related to the purchase of computer software or hardware. Kaplan and Norton's the balanced scorecard provides a method intended to give managers a fast, comprehensive view of the performance of a business.

Also, there are project portfolio management firms, such as ProSight Inc. [12], Planview [13] etc. Applications of project portfolio and resource management aim to help companies boost team performance, and enable IT management and others to access real-time data via dashboards for prioritization and rapid decision making.

Our approach is different from these business performance approaches in a number of ways. It provides a comprehensive value model that captures a multi-level model of value drivers associated business activities and components. Also, it departs from the traditional business value analysis, and employs a component-based model of business, which facilitates solution matching for business and IT alignment. Finally, it integrates portfolio optimization technology and value driver tree analysis to maximize the value of IT investments.

\section{Concluding Remarks}

Realization of value-based IT project portfolio management requires an end-to-end approach to identifying business components and IT shortfalls associated with business pain points; and modeling to capture and represent relationships among business components and activities, metrics, value drivers and IT solutions. This paper presented a value-oriented IT project portfolio management methodology which correlates business benefits with business processes and also with solutions, measuring and tracking value, and delivering value through business processes.

This approach consists of four modeling elements. First, component business modeling provides a new view of business, and helps understand the businesses pain points and identify gaps for transformation opportunities. Second, a solution catalog presents a set of complete business solutions for an industry in a process-oriented way. It enables to discover suitable solutions for the identified gaps and assess their impact on value drivers. Third, the value driver tree specifies multiple levels of strategic value drivers, business value drivers and operational value drivers. Finally, the project portfolio optimization selects one or more possible project candidates that will generate the greatest possible risk-adjusted value, and reviews the current project portfolio to adjust it for maximizing financial value.

\section{References}

1. B. Kersten and C. Verhoef, IT Portfolio Management: A Banker's Perspective on IT, Cutter IT Journal 16(4), 34-40 (2003). 
2. C-L. Tseng, K.Y. Lin, and S.K. Sundararajan, Managing Cost Overrun Risk in Project Funding Allocation, Annals of Operations Research 135(1), 127-153 (2005).

3. Y-H. Kwak and C.W. Ibbs, Assessing Organization's Project Management Maturity, Project Management Journal 31(1), 32-43 (2000).

4. N.P. Archer and F. Ghasemzadeh, An Integrated Framework for Project Portfolio Selection, International Journal of Project Management 17(4), 207-216 (1999).

5. H. M. Hess, Aligning Technology and Business: Applying Patterns for Legacy Transformation, IBM Systems Journal 44(1), 35-44, (2005).

6. L. Cherbakov, G. Galambos, R. Harishankar, S. Kalyana, and G. Rackham, Impact of Service Orientation at the Business Level, IBM Systems Journal 44(4), 653-668 (2005).

7. H. Kellerer, U. Pferschy, and D. Pisinger, Knapsack Problems (Springer, 2004).

8. Harry Markowitz, Portfolio Selection, Journal of Finance 7(1), 77-91 (1952).

9. C. Symons, The Balanced Scorecard For IT: Value Metrics, Forrester Report, November 15,2004, p. 10.

10. T. Otake and K.J. Min, Inventory and Investment in Quality Improvement Under Return on Investment Maximization, Computers \& Operations Research 28, 997-1012 (2001).

11.T. Pisello and P.A. Strassmann, IT Value Chain Management - Maximizing the ROI from IT Investments (The Information Economics Press, New Canaan, 2003).

12. ProSight (January 23, 2005); http://www.prosight.com/.

13. Planview (January 23, 2005); http://www.planview.com/. 